\title{
$\gamma$-Ray and Cosmic Ray Escape in Intensely Star-Forming Systems
}

\section{Tova M. Yoast-Hull*}

Department of Physics, University of Wisconsin-Madison

E-mail: yoasthuLIewisc.edu

\section{John S. Gallagher, III}

Department of Astronomy, University of Wisconsin-Madison

E-mail: isqdastro.wisc.edu

\section{Ellen G. Zweibel}

Departments of Astronomy \& Physics, University of Wisconsin-Madison

E-mail: zweibeldastro.wisc.edu

\begin{abstract}
Regions of intense star-formation naturally generate high number densities of cosmic rays and as such, they are of particular interest as potential contributors to the extragalactic gamma-ray background (EGB) and as potential sources of very high-energy cosmic rays (VHECRs). While models of the starburst contribution to the EGB often assume cosmic rays are confined in starbursts, cosmic rays must escape from these galaxies if they contribute to the spectrum of VHECRs as observed at Earth. The conditions in star-forming galaxies which are responsible for such high cosmic-ray injection rates also lead to large gamma-ray fluxes, except in the case of Compton thick systems where the highest energy photons are prevented from escaping. To address these contrasting ideas, we model the gamma-ray fluxes from galaxies where cosmic rays are confined and from galaxies with strong galactic winds and explore the relationship between cosmic-ray confinement and gamma-ray absorption. We present results for the nearby starburst galaxy M82 and the ultraluminous infrared galaxy Arp 220 as examples.
\end{abstract}

The 34th International Cosmic Ray Conference,

30 July- 6 August, 2015

The Hague, The Netherlands

\footnotetext{
* Speaker.
} 


\section{Introduction}

Star-forming galaxies are thought to make a significant contribution to the extragalactic gammaray background [1,2] and are being considered as potential source of high-energy neutrinos [3]. Within the subset of star-forming galaxies, starburst galaxies are the most interesting as their combination of high star-formation rates and large reservoirs of dense, molecular gas provide an excellent environment for both $\gamma$-ray and neutrino production. Because the average gas density in these systems is so large, starburst galaxies are often assumed to be proton calorimeters such that all cosmic-ray protons accelerated within the region are confined to the galaxy and thus are converted to $\gamma$-ray and neutrinos, see e.g. [4]. Here, we test whether this assumption of proton calorimetry is valid in the most extreme star-forming systems and whether the resulting $\gamma$-rays can escape.

\section{Interaction Models}

We have developed and tested a single-zone, semi-analytical (YEGZ) model for cosmic ray interactions in star-forming environments $[5,6,7,8]$. The model is specifically designed for galactic central molecular zones (CMZs) where diffusion is negligible in comparison to energy and advective losses. Assuming supernova remnants as the primary accelerators of cosmic rays in galaxies, the primary cosmic ray populations can be calculated from the observed supernova rate, infrared luminosity, and molecular gas content. Ionization, pion production, bremsstrahlung, inverse Compton, and synchrotron are included as energy loss mechanisms, and advection via a galactic wind is included as particle escape. Secondary electrons and positrons created in charged pion decay are added to the primary cosmic ray populations. From the total cosmic ray spectra, we calculate the non-thermal radio spectrum and the $\gamma$-ray flux from bremsstrahlung, inverse Compton, and neutral pion decay.

Here, we are specifically investigating the fraction of escaping cosmic-ray protons and the opacity of the interstellar medium (ISM) to $\gamma$-rays. For a given supernova rate and ISM density, we compute the $\gamma$-ray spectra produced by a range of cosmic-ray proton populations by varying the advective timescale or the wind speed. With $\chi^{2}$ tests, we compare the modeled $\gamma$-ray spectra with the observed flux, and for models within $3 \sigma$ of the best-fitting model, we calculate the escape fraction, by comparing the advective and energy-loss timescales, and the $\gamma-\gamma$ opacity. As an additional constraint, we also model radio spectrum and compare with observations to estimate the magnetic field strength.

\section{Cosmic Ray Calorimetry}

We have completed $\chi^{2}$ tests for both the radio and $\gamma$-rays in the nearby starburst galaxies M82 and NGC 253 [5, 7]. Typical wind speeds for the best-fitting models range from $200-900 \mathrm{~km} \mathrm{~s}^{-1}$ in M82 and 100-400 and $1400 \mathrm{~km} \mathrm{~s}^{-1}$ in NGC 253, depending on the initial spectral index and cosmic ray acceleration efficiency, see [9]. These wind speeds translate to advective timescales of $1.1-4.9 \times 10^{5} \mathrm{yr}$ in M82 and NGC 253, with a minimum of $3.5 \times 10^{4} \mathrm{yr}$ in NGC 253 for an acceleration efficiency of $10 \%$ (see Figure 1). Comparing these timescales to the total energy loss lifetimes, we find that M82 is $\sim 35-70 \%$ proton calorimeter and NGC 253 is a $\sim 30-85 \%$ proton 


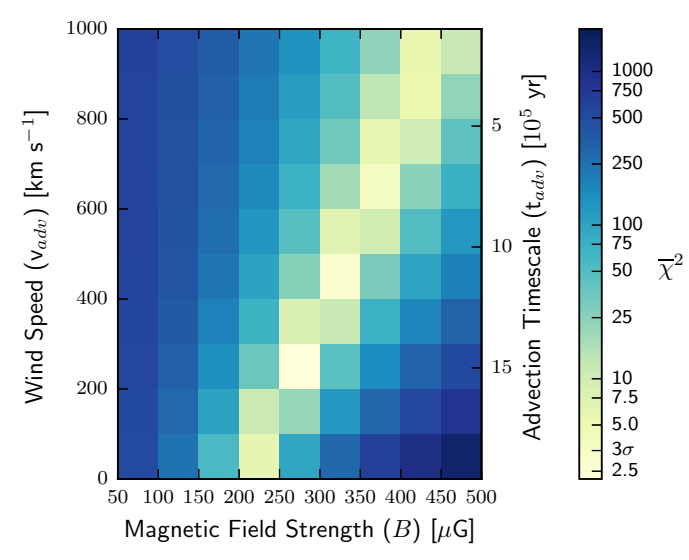

(a) M82

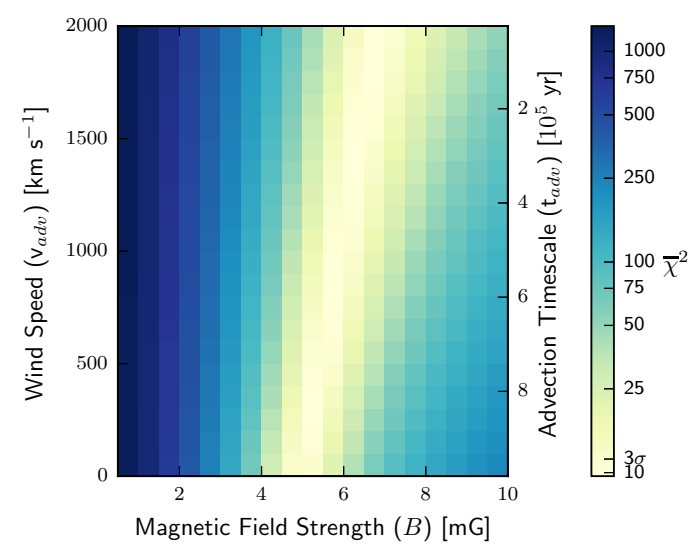

(c) $\operatorname{Arp} 220 \mathrm{E}$

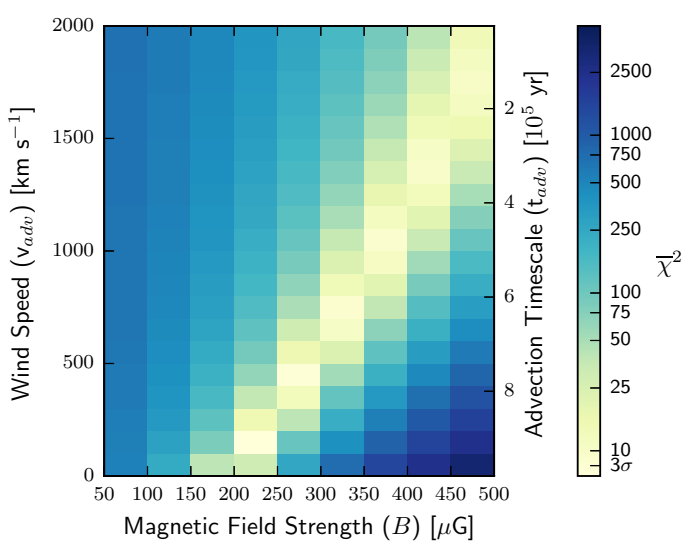

(b) NGC 253

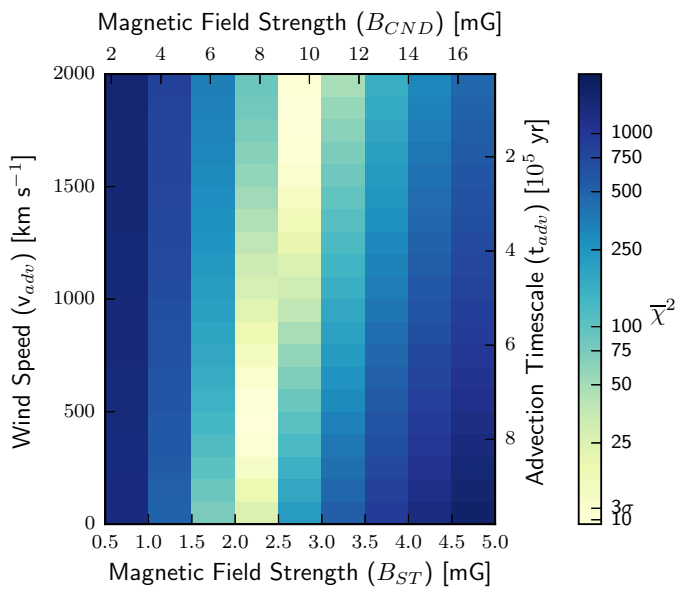

(d) $\operatorname{Arp} 220 \mathrm{~W}$

Figure 1: Contours of $\chi^{2}$ demonstrate that changes in wind speed have a major impact on the goodness of fit to the radio and $\gamma$-ray spectra for M82 and NGC 253 and little to no impact on the goodness of fit to the radio spectrum for the nuclei of Arp 220. The calorimetry fraction and the advection timescale both decrease with increasing wind speed.

calorimeter [5, 7]. As such, roughly half of the cosmic rays of $\mathrm{GeV}$ to $\mathrm{PeV}$ energies escape from these starburst systems.

While we have both radio and $\gamma$-ray observations of M82 and NGC 253, Arp 220, a nearby ultraluminous infrared galaxy (ULIRG), has yet to be detected in $\gamma$-rays. As such, we must make do with $\chi^{2}$ tests for only the radio spectrum [8]. We test models with wind speeds from 0 to 2000 $\mathrm{km} \mathrm{s}^{-1}$ and find that all tested wind speeds result in good fits for both the eastern and western nuclei in Arp 220 (see Figure 1). For both nuclei, the maximum wind speed translates to a minimum advection timescale of $2.0 \times 10^{4} \mathrm{yr}$, and thus, the eastern nucleus is a $\sim 65-100 \%$ proton 


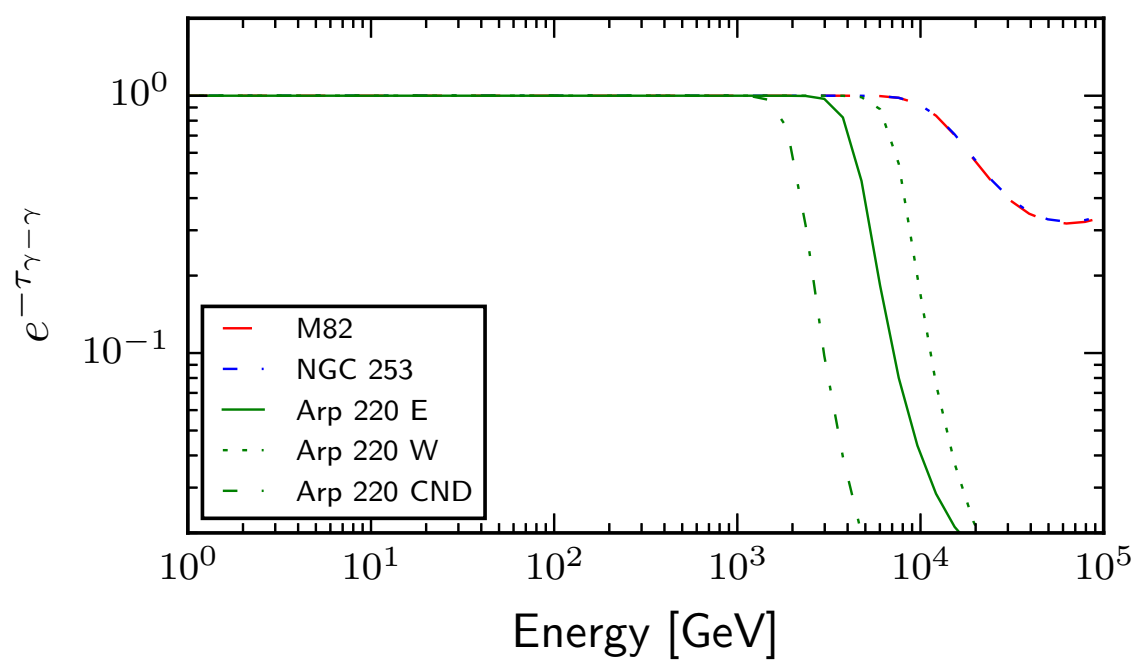

Figure 2: Optical depth $\left(\tau_{\gamma-\gamma}\right)$ is dependent upon the energy density in ambient radiation $\left(U_{\text {rad }}\right)$, the temperature or energy of the ambient photons, and the energy of the emitted $\gamma$-rays, see [10]. As demonstrated in this figure, the intense radiation fields in starburst galaxies $\left(U_{\text {rad }} \sim 10^{3}-10^{5}\right)$ absorb a large fraction of $\gamma$-rays at energies above $\sim 1-10 \mathrm{TeV}$.

calorimeter and the center of the western nucleus is a $\sim 90-100 \%$ proton calorimeter [8]. Hence, in the case of the highest advection times or lowest wind speeds, essentially no cosmic rays escape from the system.

\section{4. $\gamma$-Ray Absorption}

In addition to determining the fraction of cosmic rays which escape in intensely star-forming systems, we also examine $\gamma$-ray escape efficiencies. In intense radiation fields, $\gamma$-rays can interact with the ambient photons to produce electron-positron pairs [10,11]. Thus, these interactions result in an absorption of high-energy $\gamma$-rays. We therefore calculate the optical depth for $\gamma$-rays in M82, NGC 253, and Arp 220 (see Figure 2). For galaxies with moderate far-infrared radiation fields $\left(U_{\mathrm{rad}} \sim 500 \mathrm{eV} \mathrm{cm}^{-3}\right)$ such as M82 and NGC 253, the transparency of the ISM to $\gamma$-rays decreases significantly above $\sim 10 \mathrm{TeV}$. At energies of $5-7 \mathrm{TeV}$, a minimum of $\sim 30 \%$ of $\gamma$-rays escape from the CMZ of M82 and NGC 253. In the nuclei of Arp $220\left(U_{\mathrm{rad}} \sim 10^{4}-10^{5} \mathrm{eV} \mathrm{cm}^{-3}\right)$, transparency drops off much more quickly, beginning at $\sim 1-5 \mathrm{TeV}$, with less than $2 \%$ of $\gamma$-rays escaping from the CMZ by $10 \mathrm{TeV}$.

\section{Conclusions}

Using our cosmic-ray interaction model for starburst galaxies, we calculated the escape fraction for both cosmic rays and $\gamma$-rays from nearby starburst galaxies. We find that for nearby starburst galaxies, such as M82 and NGC 253, roughly half of cosmic-ray protons escape via galactic 
winds and thus, these types of systems are only partial proton calorimeters. For the most intense star-forming systems, such as Arp 220, the starburst zones are largely calorimetric (65-100\%), meaning that only a small fraction of cosmic rays escape. In all cases, we find that $\gamma$-rays, at energies above $\sim 10 \mathrm{TeV}$, are absorbed in the high radiation fields present in starburst galaxies. Thus, the fraction of TeV energy $\gamma$-rays escaping the system is $\sim 30 \%$ in M82 and NGC 253 and less than $\sim 2 \%$ in Arp 220 .

\section{References}

[1] T. A. Thompson, E. Quataert, and E. Waxman, The Starburst Contribution to the Extragalactic $\gamma$-Ray Background, ApJ 654 (Jan., 2007) 219-225, [astro-ph/0606665].

[2] M. Ackermann, M. Ajello, A. Allafort, L. Baldini, J. Ballet, D. Bastieri, K. Bechtol, R. Bellazzini, B. Berenji, E. D. Bloom, and et al., GeV Observations of Star-forming Galaxies with the Fermi Large Area Telescope, ApJ 755 (Aug., 2012) 164, [arXiv:1206.1346].

[3] K. Murase, M. Ahlers, and B. C. Lacki, Testing the Hadronuclear Origin of PeV Neutrinos Observed with IceCube, PhRevD 88 (Dec., 2013) 121301, [arXiv:1306.3417].

[4] A. Loeb and E. Waxman, The Cumulative Background of High Energy Neutrinos from Starburst Galaxies, JCAP 5 (May, 2006) 3, [astro-ph/0601695].

[5] T. M. Yoast-Hull, J. E. Everett, J. S. Gallagher, III, and E. G. Zweibel, Winds, Clumps, and Interacting Cosmic Rays in M82, ApJ 768 (May, 2013) 53, [arXiv: 1303.4305].

[6] T. M. Yoast-Hull, J. S. Gallagher, III, and E. G. Zweibel, The Cosmic-Ray Population of the Galactic Central Molecular Zone, ApJ 790 (Aug., 2014) 86, [arXiv: 1405.7059].

[7] T. M. Yoast-Hull, J. S. Gallagher, III, E. G. Zweibel, and J. E. Everett, Active Galactic Nuclei, Neutrinos, and Interacting Cosmic Rays in NGC 253 and NGC 1068, ApJ 780 (Jan., 2014) 137, [arXiv: 131].5586].

[8] T. M. Yoast-Hull, J. S. Gallagher, III, and E. G. Zweibel, Cosmic Rays, $\gamma$-Rays, \& Neutrinos in the Starburst Nuclei of Arp 220, MNRAS (June, 2015) [arXiv:1506.0513].

[9] T. M. Yoast-Hull, J. S. Gallagher, III, and E. G. Zweibel, in preparation (2015).

[10] C. D. Dermer and G. Menon, High Energy Radiation from Black Holes: Gamma Rays, Cosmic Rays, and Neutrinos. Princeton Univ. Press, Princeton, NJ, 2009.

[11] M. Boettcher, D. E. Harris, and H. Krawczynski, Relativistic Jets from Active Galactic Nuclei. Wiley, Berlin, Jan., 2012. 